\title{
Research on the Planning and Design of Green Residential Districts in the Process of New Urbanization
}

\author{
Di Wang ${ }^{1, a}$ \\ ${ }^{1}$ School of Highway, Chang'an University, Xi'an, 710064, China
}

\begin{abstract}
Keywords: Green residential district, Planning and design, New urbanization
\end{abstract}
\begin{abstract}
In recent years, the new urbanization has become a national strategy in China. The connotation of new urbanization lies in improving the quality of urban construction, which is compatible with the concept of green residential districts construction. This paper analyzes the impact of the new urbanization on the planning and design of green residential districts and points out the direction and the principles of the design of the green residential districts under the background of the new urbanization to provide some references for the relative researchers.
\end{abstract}

\section{Introduction}

New urbanization focuses on building a beautiful and environmentally friendly city. At the same time, it proposes that the ecological civilization should be integrated into the whole process of urbanization to take the road of intensive, intelligent, green and low-carbon urbanization. This time the green residential model introduced to everyone, and can be applied to real estate development, application to the development of new towns to go, in line with the requirements of the state. Green residential focus is placed on the development of green society. Green residential area is not only the model to create a green, environmentally friendly, the principle of sustainable development in residential area in the material space, more important is to lay the foundation for the creation of social life and the development of green. In the introduction of green residential areas, we focus on both the living area on this basis, because the residential area is an integral part of the ecological city, with urban functions. Through the construction of green residential areas can achieve the best organization of public life, enjoy the urban civilization, the ecological environment of the city to create a comfortable environment. A large number of social goals and cultural services cannot be achieved through the residential monomer, and the level of green residential areas will be concerned about the expansion from the architectural level to the community level, expanded to the development of new urban nodes. Therefore, the field of research, the study of more abundant resources, so that we can achieve a better embodiment of energy-saving emission reduction, environmental protection, land use, etc. It can achieve the best results. Green residential area model is an important tool for the development of new urbanization.

\section{Planning Directions of Green Residential Districts in the Process of Urbanization}

Green Residential Districts of Low Cost. The construction planning cannot be divorced from time and place but, in order to save resources and energy, reduce the pressure on the environmental load of building is the premise of use can promote the benign cycle of ecological system, no environmental pollution, high efficiency and energy saving construction scheme and technology, providing a healthy and safe and comfortable for the disabled. Living environment and living space, and ultimately achieve coexistence, harmonious environment and sustainable development, which is always our goal and pursuit of design. For the planning designers on the low cost of green residential area as the main research direction, familiar with the relevant knowledge and skills of cost control, with the local planning and design and technology strategy. The materials and equipment are suitable to solve problems to strive to not increase or even reduce construction costs, while achieving the goal of green residential district. At the beginning of the design, the owner only required to meet the requirements of energy saving calculation, do not do any other ecological energy-saving technology. 
In order to achieve the purpose of green building, it not only comes from the technical point of view, simply constitute a combination of architecture and technology, beginning in the design should consider environmental factors on architecture and environment to the overall consideration. Design is a very useful exploration. We avoid the surface only to explore the meaning of green building, but from the venue, layout, structure, modeling, and many other aspects of the small start. Under the condition that the cost of construction is not sufficient, the design and construction methods of rough and straight line are avoided. We strive to combine the local natural conditions, the surrounding conditions of the site, the special requirements of the construction, the low energy consumption of the later maintenance. We have a high technology and low-cost method to combine the content of the green building and the architectural features into a good integration.

Green Residential Districts of Humanity Spirit. In the final analysis, all the building energy conservation technology is only a means. All forms of construction, residential design is only a product form. The real estate industry should provide a green, healthy, ecological and harmonious living style. Green residential area to provide products and is not only a single building or residential buildings, not only is a good living environment, but also has the rich connotation of green life, real estate is the basis of innovation and industry transformation direction. The construction of green residential area emphasizes the optimization and integration of the building energy saving system, and emphasizes the industrialization and integration of the production mode. It starts from the core needs and living feelings of the residents and consumers, and actively pays attention to the change of people's needs and living style. Green residential construction, not for the green, but not for residential area with a green label, green residential area covers the living environment of resource utilization, open block, pedestrian traffic, commercial layout, cultural innovation, community diversity, and provides employment opportunities for sustainable development, in order to make residential development project organically integrated into the city. We in the construction of the community, to encourage activities, outdoor activities will increase exchanges, and therefore we require the planning of the space to be emotional, suitable for the relative crowd. We emphasize the humanistic harmony is to establish a community service system. Green residential construction has been in the past from the simple use of green technology to come out of the framework, from the narrow green technology to the broad prospects of the transformation of the real estate. The current residential planning system contains the elements of social culture, environmental development, and so on, to achieve a comprehensive transformation from the production of products to the construction of a green lifestyle, and promote the comprehensive and healthy development of green residential areas in China.

Green Residential Districts of Elderly People. Aging is an unavoidable problem of new urbanization in china. Different people have different pension model, different levels and different towns have different types of settlements. For example, in large and medium-sized city, the use of existing buildings is aging, green transformation is a comprehensive benefit in small towns and rural areas, we should strictly control the scale of real estate development projects, especially in all kinds of leisure tourism as the theme, the pension real estate development. This type of project must establish and strictly enforce the ecological planning mandatory. It should be the implementation of green building standards are higher, this is one of the effective means to prevent the excessive development of behavior, to protect the ecological environment. In the choice of the community environment, we hope to choose a clear, quiet and beautiful environment. Emphasize the spatial orientation in the community in order to improve the recognition of residential buildings by making the appearance and difference of the external environment, a sense of security. The traffic accessibility has the appropriate treatment, and strict separation of people and vehicles. In the source area also see the connection between the construction of the wind and rain corridor cannot be separated from the rain shelter between buildings. The design diversity can be suitable for different needs of the elderly. The details of the design for the elderly to consider: the area of the barrier free ramp, entrance stairs and stairs designed specifically for the physical form of the elderly, slope slowdown, width increase, set handrails. Bedroom doors are moderately relaxed scale, so that wheelchair access, to meet the needs of nursing, but also facilitate the entry and exit of the stretcher to 
ensure emergency, and do not set the threshold, no height difference. The indoor lamp switch and key position are reduced, the position of the socket is increased, and the convenience of use is increased.

\section{Design Principles of Green Residential Districts in the Process of Urbanization}

Highlight Landscaping Effect. Residential landscape should improve the greening rate to improve the living environment of people, at the same time, through the reasonable collocation of plants to show the beauty of the landscape. In the plant, specific application should make full use of trees and shrubs and herbaceous plants to improve the rate of greening, different types of plants collocation can increase the overall sense of the level, the formation of a layered plant greening effect, give people a feeling of trees at the same time, can choose the appropriate color leafed plants, use of plant color to beautify the environment effect of improved single color in the landscape. Shrub plants reflect the rhythm of the plant landscape. It can achieve a good decorative effect. In order to achieve better results, we can also use orchids. The special flavor of the plant creates a livable environment, which gives people a comfortable spiritual enjoyment. Some communities design a series of stone reliefs on the periphery of the landscape, showing the cultural atmosphere of the community. Green bamboo and azaleas and other plants to fully demonstrate the characteristics of the community in the landscape, rich residential landscape environment, people have a sense of immediate, and create a good landscape effect. In the area outside the green design, should reflect their own style, making the overall landscape have certain characteristics, avoid monotony in addition to conventional green plants, can be integrated into other elements, change the style characteristics of landscape. We can use the sculpture and other materials to create the landscape, plus a variety of plant embellishment, so that the residential area has a strong sense of the external landscape and modern art. At the same time, personalized landscape design is also a business card, can not only give people a deep impression, but also play a role in the publicity community.

Select Green Materials. According to the environmental design of roof rainwater and ground rainwater collection technology, the introduction of rainwater tanks, decontamination and other physical and chemical treatment and then enter the water system using water purification, for road dust, clean water, landscaping, water supplement water use, water recycling use of this system, can save a lot of drinking water. As an ecological district, the building materials should be strictly designed, the building materials and products shall be approved by the national environmental labeling the application of environmentally friendly materials such as concrete mixing technology, application of aerated concrete brick, from the source to ensure that the construction of environmental protection. Historic buildings, historic sites, etc., also need to be preserved and inherited, retaining the local structure and local flavor. The ancient and famous trees are the product of the long history of nature and history, and the precious legacy of nature and their predecessors. It is everyone's duty to protect it. We can make full use of local materials to improve ecological benefits. Native plants are the result of natural selection, which is highly adaptable to local soil and climatic conditions. Therefore, the full use of native plants can improve the survival rate of plants, and will grow well. Landscape architecture in the use of local building materials can reduce costs, save money, but also can make the different regions of the landscape to reflect the regional characteristics. Local living material is the most convenient and desirable resources, it can be used in the landscape will produce surprising native landscape.

Enhance Cultural Connotations. For the modern residential landscape, people are not only limited to the pursuit of greening effect, but also pay attention to the expression of cultural connotation in the residential environment. This can be created through a variety of landscape design, for example, into a certain cultural landscape, to a certain extent, to achieve this effect. Especially in the area of some important position, the design of some representative garden sculpture, sketch, etc., can be a good performance of the overall style of the district characteristics. Residential green space is closely linked with people's daily life, so the landscape should pay attention to the design and creation of space and the application of plants to create a suitable space for people's activities. Specifically, the use of high and low density of plants, such as the formation of open, semi closed, 
fully enclosed space to meet the needs of people's activities. The space composed of tall trees and some shrubs gives people a feeling of concealment and security, which is suitable for people to communicate with each other. It is composed of low shrubs, herbs, grass and so on. It has a wide field of vision, which is convenient for the elderly and children to do some outdoor activities. In the process of ecological living area landscape plant configuration, should be conducive to the formation of open pattern, layout of cultural and recreational facilities and leisure sports exchange humanization space and facility integration in the landscape. The building is conducive to the development of social public space. The unique cultural and artistic taste of landscape garden features and rich local color, can enhance the ecological community image, at the same time, green residential area landscape can provide cultural and leisure activities for the residents and public convenience, but also can make the residents get the feeling of beauty and good lessons.

\section{Conclusion}

The new urbanization construction is being pushed forward, which brings many new subjects to the planning and design of green residential districts. The planning of green residential districts should meet the needs of the new urbanization. The design of green residential districts should reflect the concept of new urbanization. Under the background of new urbanization, the theory of the planning and design of green residential districts should be improved and enriched in practice.

\section{References}

[1] Wan Min, Wu Can. Planning and Design Research about Green Axle-type Residential District [J]. Chinese Landscape Architecture, 2012(9): 39-43.

[2] Yuan Ling. On Green Community Planning and Design in the Context of New Urbanization [J]. Design Community, 2016(2): 109-115.

[3] Wu Cuifeng. Discussion on ecological green design of residential district [J]. Shanxi Architecture, 2012, 38(30): 21-22.

[4] Wang Yu, Landscape Design of Green Spaces in Residential Districts [J]. Journal of Anhui Agricultural Sciences, 2012, 40(3): 1551-1553.

姓名: 王迪

单位: 长安大学公路学院

地址：陕西省西安市雁塔区南二环路中段长安大学本部交通科技大厦905办公室王迪（收）

邮编: 710064

电话: 13619286573 